hunting by sight. There were more confirmatory data to support the validity of this hypothesis, particularly of the kind that correlates cyclomorphic phenomena with the presence of fish. In addition, it has been nicely shown that the visual acuity of planktiverous fish is of the order expected by the hypothesis so that reducing apparent size is a valid strategy for plankters. There is consensus that predation is, indeed, the selective force behind the cyclomorphosis of the larger cladocerans.

Attention has now shifted to the adaptive responses produced by small invertebrate predators. Some of these are at low Reynold's number like their prey, and have comparatively low success rates. Others, like Chaoborus, can cross the Reynold's number divide, make fast capture movements, and wait in ambush on vertical migration paths. The rotifer Asplanchna, an animal not much larger than the other rotifers on which it preys, is most successful against victims that are not spiny, suggesting that spines are an adaptation to Asplanchna predation. Heterecope predation can induce the possession of spines in prey populations rather as fish predation induces cyclomorphosis in Cladocera. There was a general feeling at the conference that small predators may be more important in producing structure in zooplankton communities than competition between herbivores.

With filtering, vertical migration, and cyclomorphosis becoming understood we may claim to be mastering some of the immmediate causes of community structure in the plankton. The underlying causes remain obscure. The difficulty in deciding even whether responses involve selection or merely phenotypic plasticity can be gauged by the fact that there still seems uncertainty about what constitutes many planktonic species. Taxonomists hesitate to put names on tropical zooplankters, not feeling sure whether many of these can be fairly assigned to species known from temperate latitudes, and there seems a growing doubt that species from temperate Europe and North America are really the same even though they look extremely similar. A further question is how much of the response to selective pressures such as predation can be genetic when typical populations are asexual clones?

One particular peculiarity was called to mind by several observations from the tropics: there are usually very few species of zooplankter in the open water of a tropical lake, perhaps one or two copepods, one or two cladocerans, and a few rotifers. This comparatively tiny assemblage of herbivores feeds on an array of phytoplankton

\title{
Magnetics, climate and eccentricity
}

\section{from Peter J. Smith}

ONE of the most surprising correlations to have been discovered in recent years is that between fluctuations in palaeoclimate and the palaeomagnetic field--surprising because it is difficult to imagine any possible causal connection between an atmospheric phenomenon and motions in the Earth's fluid outer core. Indeed, so astonishing was the geomagnetismclimate relationship that, when its existence was first hinted at, a good many people were inclined not to believe it. But if there are still any sceptics around, it may be presumed that they never saw the report by Wollin et al. (Geophys. Res. Lett. 4, 267 ; 1977) published some 18 months or so ago. For in it Wollin and his colleagues presented data from several deep sea sediment cores, showing a convincing general (anti) correlation between the intensity of core magnetisation and foraminiferal abundance-which means a correlation between warm climatic stages and periods of low geomagnetic field intensity.

They went even further. Harrison (Earth-Sci. Rev. 10, 1; 1974) and Kent and Opdyke (EOS 57, 237; 1976) had already suggested that any relationship between geomagnetic field and climate may be the result of a third phenomenon; and Hays et al. (Science 194, 1121; 1976) had linked ice ages with the obliquity and precession of the Earth's orbit. Wollin and his coworkers therefore plotted not only magnetisation and foraminiferal abundance but also the variation in the Earth's orbital eccentricity as deduced by Van Woerkom (in Climatic Change (ed. Shapley, H.) Harvard University Press, 1953). A threefold correlation between warm climate, low field intensity and high orbital eccentricity for the past $0.8 \mathrm{Myr}$ was thus revealed, the clear implication being that eccentricity modulates both climate and geomagnetic field.

In view (presumably) of previous scepticism on the climate-

species that may number 200 or so different kinds. On land, such an array of plants always seems to be under pressure from many different herbivores, and we call on the "cropping principle' to explain plant diversity. It is an apparent paradox of the zooplankton that natural selection has not provided an array of herbivore species to specialise on the diverse array of geomagnetism issue, Wollin et al. were rather cautious about the reality of the relationship emerging from their data. But if their new results are anything to go by they need not have worried, for they have now demonstrated a similar correlation in a single North Atlantic sediment core spanning no less than the whole of the past 2 million years (Earth planet. Sci. Lett. 41, 395; 1978). This time they used the more recent eccentricity data from Vernekar (Meteorol. Monograph 12, American Meteorological Society, 1972), but the result was the same. Warm climate matches low field matches high eccentricity.

But why? For the time being that is anyone's guess; so Wollin and his colleagues have had a go at it themselves. They begin with the premise that because the density of the Earth's core is greater than that of the nearsurface zone, the core-mantle boundary is less elliptical than the Earth's surface. They then argue that, as a result, the torque exerted by solar and lunar gravity on the core is smaller than that on the mantle; so the core tends to precess more slowly than the mantle. Moreover, as the Earth's orbit is eccentric, the solar gravitational field acting on the Earth has an annual variation that increases as the eccentricity increases. Therefore when the eccentricity is greater, the difference between the torques acting on mantle and core is greater, and the tendency of the core and mantle to precess at different rates is enhanced.

Wollin et al. then go on to suggest that such an increase in the differential procession will lead to perturbations in the core's convective flow of such a type as to reduce the strength of the geomagnetic dipole and hence reduce the effectiveness of the magnetic shield against corpuscular radiation. Anyone else want to try?

Peter J. Smith is a Reader in the Department of Earth Sciences, The Open University, Milton Keynes.

plants. Probably the answer to this paradox lies in the fact that the discriminatory powers of planktonic herbivores, so clearly revealed at this conference, are yet rudimentary compared with those of small terrestrial herbivores.

Paul Colinvaux is in the Department of Zoology, Ohio State University. 